\title{
Diversidad de helechos y licófitas de la Reserva Natural Punta Lara, Buenos Aires, Argentina
}

\author{
Gabriela E. Giudice ${ }^{1}$, Juan Pablo Ramos Giacosa ${ }^{1,2}$, María Luján Luna ${ }^{1,3}$, Agustina Yañez ${ }^{1}$ \& \\ Elías R. de la Sota ${ }^{1,2}$ \\ 1. Facultad de Ciencias Naturales y Museo, Universidad Nacional de La Plata. Paseo del Bosque s/nº, 1900 La Plata, \\ Argentina; gegiudice@hotmail.com,yanez_agustina@hotmail.com,sota@fcnym.unlp.edu.ar \\ 2. Consejo Nacional de Investigaciones Científicas y Técnicas, Avda. Rivadavia 1917 - CP C1033AAJ - Ciudad de \\ Buenos Aires, Argentina; jpramosgiacosa@hotmail.com \\ 3. Comisión de Investigaciones Científicas de la Provincia de Buenos Aires, Camino Gral. Belgrano y 526, CP 1900, La \\ Plata, Argentina; lujanluna@fcnym.unlp.edu.ar
}

Recibido 13-IX-2010. Corregido 16-XII-2010. Aceptado 24-I-2011.

\begin{abstract}
Diversity of ferns and lycophytes from Reserva Natural Punta Lara, Buenos Aires, Argentina. Punta Lara Natural Reserve shelters the relict of the Southernmost riparian forest in the world, where the flora is threatened by the anthropic expansion and by the introduction of exotic species. In this area the ferns and lycophytes grow in particular environments as marshland, grassland and marginal forest. The aim of this work was to evaluate the diversity of ferns and lycophytes that inhabit in Punta Lara Natural Reserve. Field trips were conducted from 2006 to 2009 and local herbaria and specific bibliography were also revised. As a result of our study twenty four taxa of ferns and lycophytes were recognized. Their habit was mainly terrestrial (70\%), fewer aquatic (17\%) and epiphytic (13\%). Five native taxa were scarcely recorded whereas two native species previously documented were not found. We assume that the main threats on ferns and lycophytes populations in Punta Lara Reserve are: removal for comercialization, invasion by exotic species and climatic fluctuations (drought periods). Keys for taxa identification are given and strategies for the conservation of threatened taxa are proposed. Rev. Biol. Trop. 59 (3): 1037-1046. Epub 2011 September 01.
\end{abstract}

Key words: ferns, lycophytes, Argentina, Buenos Aires, Punta Lara Natural Reserve, diversity.

Los helechos y licofitas suelen ser sensibles a los cambios ambientales y a las presiones antrópicas, debido a su reproducción sexual a través de esporas, su crecimiento lento y la ausencia de estrategias competitivas agresivas (Mickel 1977).

Hasta el año 1987 el número de helechos y licofitas citados para la Reserva Punta Lara era de 30, incluyendo taxa específicos e infraespecíficos (Cabrera 1960, 1971, Capurro 1969, Moschione 1987).

La Reserva Natural Punta Lara está situada sobre la ribera argentina del Río de La Plata, $12 \mathrm{~km}$ al norte de la ciudad de La Plata (34\%47' $\left.\mathrm{S}-58^{\circ} 01^{\prime} \mathrm{W}\right)$. Comprende aproximadamente
6000 ha de ambientes ribereños entre los cuales se distinguen la costa, la selva marginal, los espinales, los pajonales inundados y los pastizales (Fig. 1 A-D). En estos ambientes crecen distintas agrupaciones de especies que se encuentran desde los márgenes del río hacia el interior del continente, según las condiciones de humedad y la composición de los suelos (Cabrera \& Dawson 1944). El clima es templado-cálido, con precipitaciones medias anuales de $1000 \mathrm{~mm}$ y una temperatura media anual de $18^{\circ} \mathrm{C}$.

Desde el punto de vista fitogeográfico la Reserva Natural Punta Lara se encuentra incluida en el Dominio Chaqueño, Provincia 

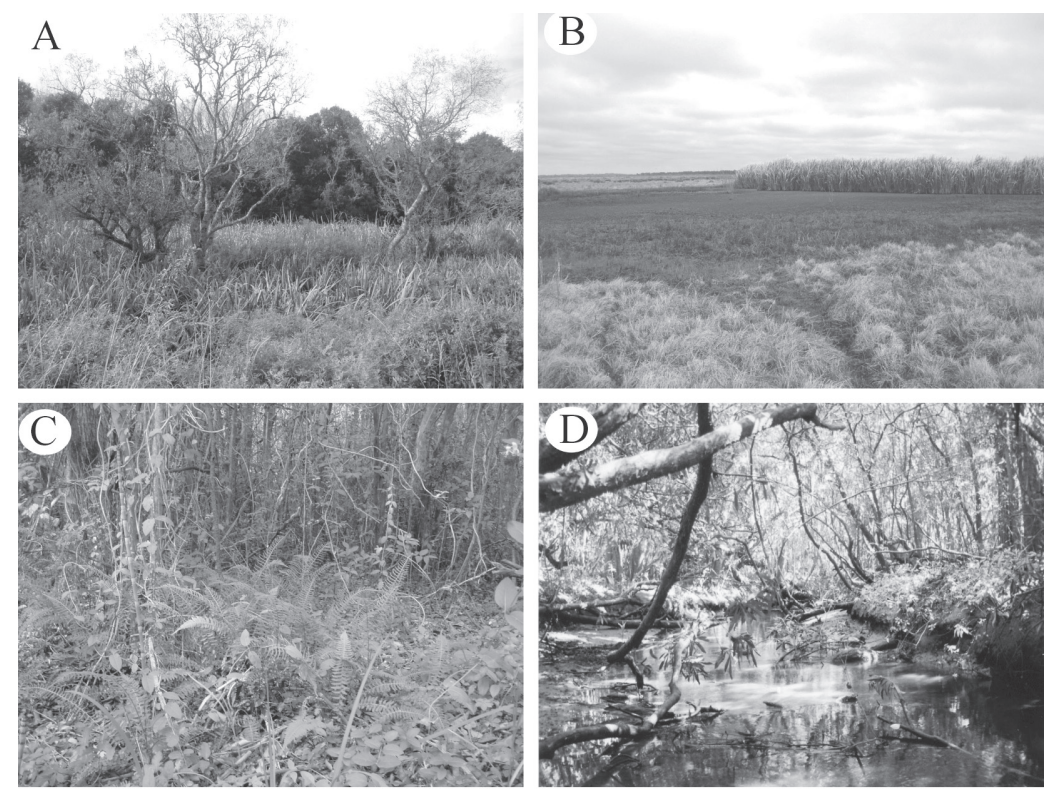

Fig. 1. Ambientes de la Reserva Natural Punta Lara. A: Pajonal. B: Pastizal. C: Selva Marginal. D: Selva Marginal, arroyo Las Cañas.

Fig. 1. Environments of Punta Lara Natural Reserve. A: Marshland. B: Grassland. C: Marginal Forest. D: Marginal Forest, Las Cañas Stream.

Pampeana y dentro de ésta en el distrito Pampeano Oriental. Las comunidades edáficas de este distrito son muy numerosas, especialmente en su borde nordeste, donde la influencia del Delta y del Río de la Plata determinan una gran variedad de nichos ecológicos diferentes. En los albardones del Delta y de la ribera platense hasta Punta Lara, existen selvas marginales hidrófilas similares a las encontradas en el Distrito Uruguayense, que se extienden por el sur de las Provincias de Entre Ríos y Santa Fe (Cabrera 1971).

En la zona de estudio, la selva marginal se desarrolla sobre albardones bordeados por pequeños arroyos que ingresan desde el río y probablemente constituyan el relicto más austral de las selvas en galerías asociadas a los ríos Paraná y Uruguay (Cabrera \& Dawson1944, Cabrera 1976).

En el ambiente de selva marginal en Punta Lara el nivel superior arbóreo está conformado por especies nativas dominantes como: laurel (Ocotea acutifolia (Nees) Mez.), chalchal
(Allophylus edulis (St. Hil.) Radlk), yerba de bugre (Lonchocarpus nitidus Vog.) Benth.), mata ojos (Pouteria salicifolia (Kunth) Baehni) y algunas especies exóticas como ligustro (Ligustrum lucidum W.T. Aiton) (Dascanio \& Ricci 1988).

Debajo de este estrato superior se desarrollan los estratos arbustivos y herbáceos, de los cuales los helechos y licofitas forman parte fundamental, creciendo entre tacuaras bravas (Guadua trinii (Ness) Ness), malvas (Pavonia malvaceae (Vell.) Krap. \& Crist.), tala gateador (Celtis iguanea (Jacq.) Sarg.) y renovales de las especies dominantes. Los helechos y licofitas se desarrollan directamente sobre el sustrato o sobre troncos de árboles caídos. Asimismo, estos grupos se encuentran representados dentro de la abundante flora epifítica, junto con las orquídeas y los claveles del aire.

Otro ambiente característico de la Reserva es el pastizal, el cual se desarrolla desde la culminación de los pajonales inundados (a un $1.5 \mathrm{~km}$ de la costa) hasta el límite SO de 
la Reserva marcado por la Autopista Buenos Aires-La Plata. El pastizal está constituido fundamentalmente por gramíneas las cuales crecen en asociación con especies herbáceas, entre ellas algunos helechos. El desarrollo de helechos en este ambiente es propiciado por la frecuente inundación de los suelos asociada a regímenes temporales de agua (Cabrera 1960).

El fuerte impacto antrópico registrado en la Provincia de Buenos Aires, la más poblada de la República Argentina (13 818677 habitantes según INDEC 2001), causado principalmente por las actividades agrícola-ganaderas y el creciente empleo de los helechos y licofitas como plantas ornamentales, han puesto en riesgo su supervivencia en la región.

Un estudio realizado por Delucchi (2006) sobre las especies vegetales amenazadas de la Provincia de Buenos Aires, considera a ocho taxa de helechos y licofitas "en peligro" y a 13 como "vulnerables" como consecuencia de la acción antrópica y la reducción del hábitat. Asimismo, el autor menciona a Microgramma vaccinifolia (Langsd. \& Fisch.) Copel, como extinta en esta Provincia.

En la Reserva Natural Punta Lara se registró en las últimas décadas un marcado ingreso de especies exóticas, como ligustro (Ligustrum lucidum), zarza mora (Rubus ulmifolius Schott), lirio amarillo (Iris pseudoacorus L.) y madreselva (Lonicera japonica Thunb.), lo cual sumado a la recolecta furtiva de especies nativas de interés ornamental representan una amenaza para las poblaciones naturales que crecen en la zona.

El objetivo de esta contribución es actualizar la información sobre la diversidad de los helechos y licofitas que habitan en la Reserva Natural Punta Lara con el fin de contribuir a la conservación de estos grupos de plantas en el relicto más austral de las selvas en galería.

\section{MATERIALES Y MÉTODOS}

Para llevar a cabo este estudio se realizaron viajes de campo durante los años 2006-2009, entre los meses de noviembre a mayo, en los cuales los helechos y licofitas se encuentran fértiles. El material colectado fue herborizado y depositado en el herbario LP. Asimismo, se recabó información a través de la consulta bibliográfica y de ejemplares de herbario de las siguientes instituciones (siglas según Holmgren et al. 1990): BA, BAA, BAB, BAF, BAL, BBB, LP, LPAG, SI.

Con los datos obtenidos se confeccionó una clave de los géneros presentes en la reserva y claves de especies para aquellos géneros con dos o más representantes.

Los aspectos nomenclaturales de las especies comprendidas en este trabajo se trataron de acuerdo a Zuloaga et al. (2008).

Material representativo estudiado:

\section{Adiantopsis chlorophylla}

Buenos Aires, Pdo. Ensenada, R N Punta Lara, albardón "La Araucaria", 7/11/2007, Ramos Giacosa 27 (LP)

\section{Adiantum capillus-veneris}

Buenos Aires, Pdo. Ensenada, R N Punta Lara, sobre paredes de casa de guardaparque, escapada de cultivo, 3/04/2007, Ramos Giacosa 8 (LP)

\section{Adiantum raddianum}

Buenos Aires, Pdo. Ensenada, R N Punta Lara, Boca Cerrada, en margen de arroyo, 3/04/2007, Ramos Giacosa 14 (LP); Idem, Punta Lara, 29/10/1946, Pérez Moreau s/n, BA 42953

\section{Anogramma chaerophylla}

Buenos Aires, Pdo. Ensenada, R N Punta Lara, bajo las pasarelas, 3/04/2007, Ramos Giacosa 7 (LP); Idem, Punta Lara, 15/8/1944, Fabris $24(\mathrm{SI})$

\section{Asplenium sellowianum}

Buenos Aires, Pdo. Ensenada, R N Punta Lara, márgenes de arroyo Las Cañas, Ramos Giacosa 13 (LP); Idem, Punta Lara, 22/8/1954, Mazzucconi 649 (BAB) 


\section{Asplenium ulbrichtii}

Buenos Aires, Pdo. Ensenada, R N Punta Lara, área del arroyo Las Cañas, 6/12/2006, Ramos Giacosa 12 (LP); Idem, 20/8/1973, Zardini 177 (LP)

\section{Azolla filiculoides}

Buenos Aires, Pdo. Ensenada, Punta Lara, 1/11/1942, Pérez Moreau s/n, BA 43381; Idem, Puesto El coronillo, 26/09/2008, Ramos Giacosa 36 (LP)

Blechnum australe subsp. auriculatum

Buenos Aires, Pdo. Ensenada, R N Punta Lara, área del arroyo Las Cañas, 6/12/2006, Ramos Giacosa 17 (LP); Idem, 15/11/1941, Dawson 1141 (SI)

\section{Ctenitis submarginalis}

Buenos Aires, Pdo. Ensenada, R N Punta Lara, arroyo Las Cañas, 6/12/2006, Ramos Giacosa 24 (LP); Idem, 7/11/2007, Ramos Giacosa 9 (LP); Idem, Punta Lara, 24/10/1937, Capurro $\mathrm{s} / \mathrm{n}, \mathrm{BA} 60849$

\section{Doryopteris concolor}

Buenos Aires, Pdo. Ensenada, R N Punta Lara, albardón La Araucaria, 7/11/2007, Ramos Giacosa $28(\mathrm{LP})$

\section{Doryopteris pentagona}

Buenos Aires, Pdo. Ensenada, R N Punta Lara, área del arroyo Las Cañas, 6/12/ 2006, Ramos Giacosa 30 (LP)

\section{Equisetum giganteum}

Buenos Aires, Pdo. Ensenada, R N Punta Lara, cerca de camino Negro, creciendo al sol, 7/11/2007, Ramos Giacosa 10 (LP), Idem, Punta Lara, 2573/1979, Freire s/n (LPAG); Idem, 10/1962, Amorín 63 (BAF); Idem, Molfino y $\mathrm{Clos} 76$ (BAB).

\section{Marsilea ancylopoda}

Buenos Aires, Pdo. Ensenada, R N Punta Lara, 26/09/2008, Puesto El Coronillo, campo de pastoreo, Ramos Giacosa 35 (LP).

\section{Microgramma mortoniana}

Buenos Aires, Pdo. Ensenada, R N Punta Lara, arroyo Las Cañas, epifita, 6/12/ 2006, Ramos Giacosa 20 (LP); Idem, 7/11/2007, Ramos Giacosa 15 (LP); Idem, camino a Boca Cerrada, 7/11/2007, Ramos Giacosa 18 (LP); Idem, Punta Lara, 4/3/1954, Calderón 432 (BAA)

\section{Nephrolepis cordifolia}

Buenos Aires, Pdo. Ensenada, R N Punta Lara, arroyo Las Cañas, 6/12/ 2006, Ramos Giacosa 22 (LP)

\section{Pleopeltis macrocarpa}

Buenos Aires, Pdo. Ensenada, R N Punta Lara, Camino Negro, suelo inundado, sobre tronco caído de Arce, 30/07/2009, Ramos Giacosa 26 (LP); Idem, zona de arroyo Las Cañas, epifito, 6/12/ 2006, Ramos Giacosa 16 (LP); Idem, Punta Lara, 4/1961, Gamerro 21678 (SI)

\section{Pleopeltis squalida}

Buenos Aires, Pdo. Ensenada, R N Punta Lara, albardón "La Araucaria", 7/11/2007, Ramos Giacosa 29 (LP)

\section{Pteris deflexa}

Buenos Aires, Pdo. Ensenada, R N Punta Lara, arroyo Las Cañas, 6/12/ 2006, Ramos Giacosa 21 (LP)

\section{Rumohra adiantiformis}

Buenos Aires, Pdo. Ensenada, R N Punta Lara, arroyo Las Cañas, 6/12/ 2006, Ramos Giacosa 23 (LP)

\section{Salvinia biloba}

Buenos Aires, Pdo. Ensenada, R N Punta Lara, 17/7/1946, Partridge s/n BA 596; Idem, 5/9/1945, Capurro 457 (LP)

\section{Salvinia minima}

Buenos Aires, Pdo. Ensenada, R N Punta Lara, 17/7/1946, Partridge s/n, BA 43516; Idem, Boca Cerrada, 4/1995, Denham \& Manchini $\mathrm{s} / \mathrm{n}(\mathrm{LP})$

\section{Selaginella muscosa}

Buenos Aires, Pdo. Ensenada, R N Punta Lara, 23/8/1936, Capurro s/n ${ }^{\circ}$, BA 56658; Idem, 
matorral ribereño, 6/8/2008, Ramos Giacosa 37 (LP)

\section{Thelypteris abbiattii}

Buenos Aires, Pdo. Ensenada, R N Punta Lara, arroyo Las Cañas, 6/12/ 2006, Ramos Giacosa 11 (LP); Idem, 20/8/1973, Zardini 176 (LP); Idem, 28/8/1966, Zonina s/n, BA 64431

\section{Thelypteris hispidula}

Buenos Aires, Pdo. Ensenada, R N Punta Lara, arroyo Las Cañas, 6/12/2006, Ramos Giacosa 25 (LP)

\section{RESULTADOS}

En este estudio se registraron para la Reserva Natural Punta Lara veinticuatro taxa de helechos y licofitas, incluyendo tres nuevas citas para el área: Doryopteris pentagona Pic. Serm. (Fig. 2A), Pleopeltis squalida (Vell.) de la Sota (Fig. 2B) y Thelypteris hispidula (Decne.) C. F. Reed. (Fig. 2C).

En esta región los grupos en estudio se desarrollan principalmente en el ambiente de selva marginal. El hábito predominante es el terrestre $(70 \%)$, se encontraron algunas
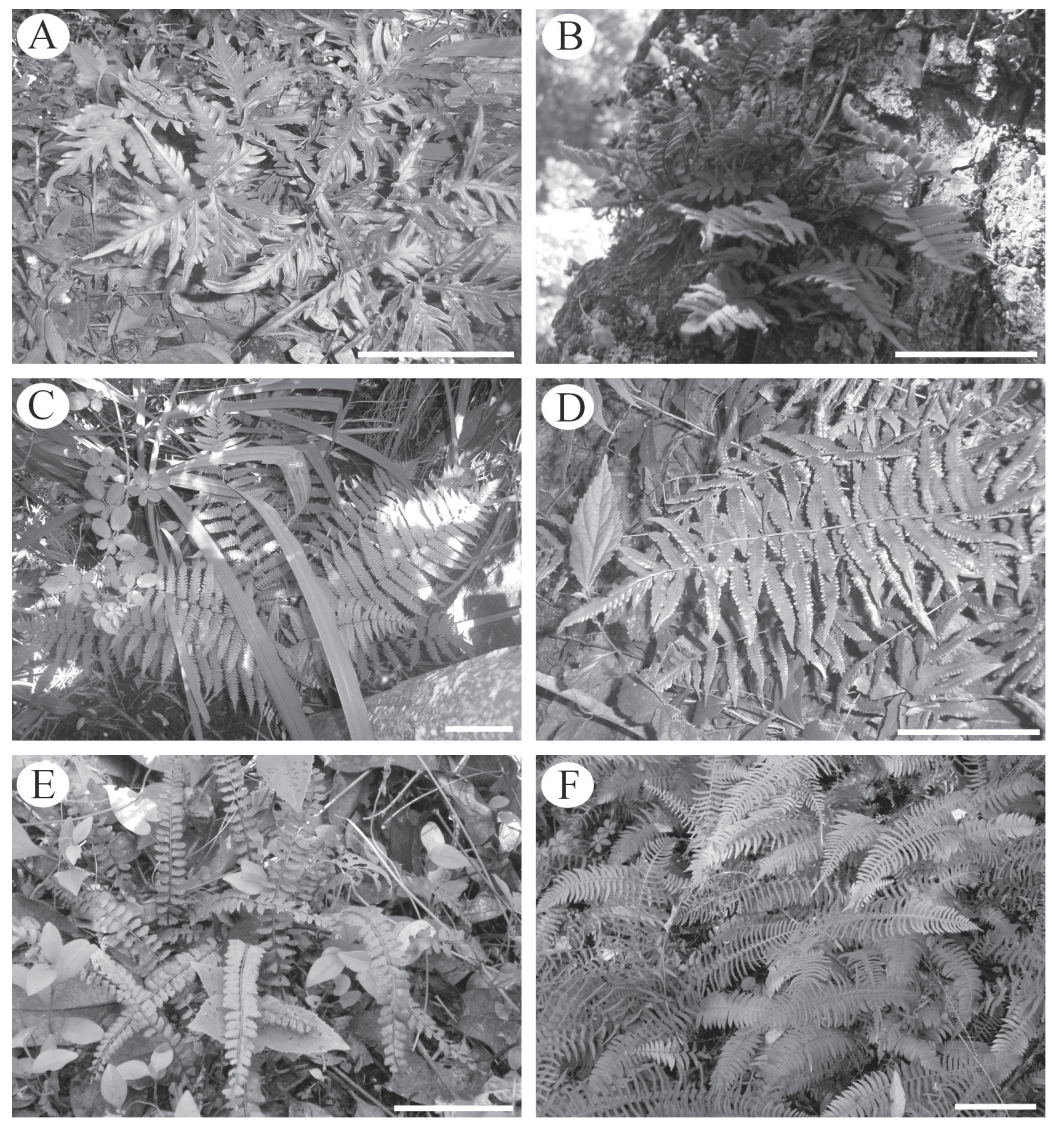

Fig. 2. Helechos de la Reserva Natural Punta Lara. A: Doryopteris pentagona. B: Pleopeltis squalida. C: Thelypteris hispidula. D. Thelypteris abbiattii. E. Asplenium ulbrichtii. F. Blechnum australe subsp. auriculatum. Escalas: A, C-F: $10 \mathrm{~cm}$. B: $5 \mathrm{~cm}$.

Fig. 2. Ferns of Punta Lara Natural Reserve. A: Doryopteris pentagona. B: Pleopeltis squalida. C: Thelypteris hispidula. D. Thelypteris abbiattii. E. Asplenium ulbrichtii. F. Blechnum australe subsp. auriculatum. Scale bars: A, C-F: $10 \mathrm{~cm}$. B: $5 \mathrm{~cm}$. 
especies acuáticas (17\%) y epifitas (13\%). Los individuos de hábito epífito fueron hallados sobre: Erythrina crista-galli L. ("ceibo"), Pouteria salicifolia (Spreng.) Radlk. ("mataojo"), Terminalia australis Camb. ("palo amarillo") y Ligustrum lucidum W. T. Aiton ("ligustro").

Los taxa registrados con mayor frecuencia fueron Thelypteris abbiattii (Fig. 2.D), Asplenium ulbrichtii (Fig. 2E), Microgramma mortoniana y Blechnum australe subsp. auriculatum (Fig. 2F). Thelypteris abbiattii abunda en los márgenes de los arroyos, en zonas frecuentemente inundadas. Asplenium ulbrichtii se caracteriza por su reproducción vegetativa a través de raquis gemíferos, por lo que a menudo forma céspedes o poblaciones conspicuas sobre troncos caídos. Blechnum australe subsp. auriculatum, con sus rizomas estoloníferos, forma poblaciones extensas en el interior de la selva. Entre las epífitas se destaca Microgramma mortoniana, la cual habita también en la selva, sobre los árboles dominantes y troncos caídos.

Algunos taxa como Pleopeltis macrocarpa, Ctenitis submarginalis, Adiantopsis chlorophylla, Adiantum raddianum, Rumohra adiantiformis y Selaginella muscosa, fueron registrados con escasa frecuencia o formando parte de poblaciones reducidas o inclusive como individuos aislados.

Pleopeltis macrocarpa, es una especie epífita que se halla habitualmente sobre "palo amarillo" y "ceibo", mientras que individuos aislados de Ctenitis submarginalis y de Rumohra adiantiformis crecen en zonas próximas al arroyo Las Cañas. En este ambiente se observó, asimismo, un único ejemplar de Doryopteris pentagona.

En la zona del albardón "La Araucaria", se identificaron individuos también aislados de Adiantopsis chlorophylla.

En lo relacionado con Adiantum raddianum, se encontraron poblaciones reducidas en la zona de "Boca Cerrada" e individuos aislados en los márgenes del arroyo Las Cañas. Poblaciones escasas de Selaginella muscosa fueron documentadas en los matorrales ribereños.

A continuación se presenta una clave para la identificación de los taxa de helechos y licofitas registrados en la Reserva Natural Punta Lara (Buenos Aires):

1. Plantas acuáticas o palustres (2)

1'. Plantas epífíticas, terrestres o saxícolas (6)

2. Plantas flotantes (3)

2'. Plantas arraigadas (4)

3. Rizoma corto con raíces aisladas, cubierto totalmente por frondes pequeñas bilobuladas. Esporocarpos dispuestos de

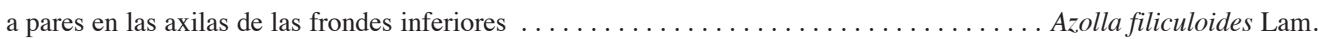

3’. Rizoma ramificado cubierto por numerosos pelos. Raíces verdaderas ausentes. Frondes con tres pinnas, dos flotantes y una sumergida dividida que porta los esporocarpos $\ldots \ldots \ldots \ldots \ldots \ldots \ldots \ldots \ldots$ Salvinia Ség

4. Plantas con ejes rastreros ramificados dicotómicamente y rizóforos ventrales. Microfilos reducidos, anisofilas ...... Selaginella muscosa Spring

4'. Plantas con ejes aéreos o sumergidos, sin rizóforos. Eufilos de diversos tamaños y formas.. . . . . . . . . . . . (5)

5. Ejes aéreos erectos, con nudos y entrenudos bien marcados, acanalados. Láminas reducidas, soldadas, de disposición verticilada. Esporangios sobre esporangióforos peltados agrupados en estróbilos terminales $\ldots \ldots \ldots \ldots \ldots \ldots$

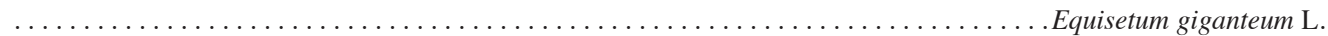

5'. Ejes sumergidos, largamente rastreros. Láminas largamente pecioladas, pinnadas, con dos pares de pinnas opuestas sobre raquis brevísimo. Macro y microsporangios agrupados en esporocarpos . . . . . Marsilea ancylopoda A. Braun

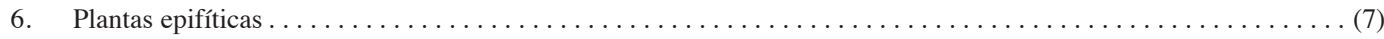

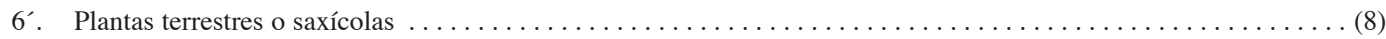

7. Frondes heteromorfas, subsésiles, glabras o con pelos pequeños . . . . . . . . Micrograma mortoniana de la Sota

$7^{\prime}$. Frondes isomorfas, pecioladas, con escamas peltadas de margen microdentado $\ldots \ldots \ldots \ldots \ldots \ldots \ldots \ldots \ldots$ $\ldots \ldots \ldots \ldots \ldots \ldots \ldots \ldots \ldots \ldots \ldots \ldots \ldots \ldots \ldots \ldots \ldots \ldots \ldots \ldots \ldots \ldots \ldots \ldots \ldots \ldots$.eoltis Humb. \& Bonpl. ex Willd.

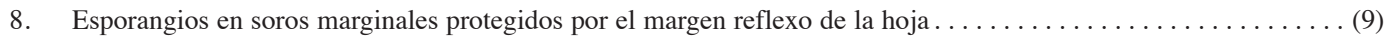

8'. Esporangios en soros de diversa ubicación, desnudos o protegidos por indusios . . . . . . . . . . . . . (12) 
9. Soros insertos sobre los márgenes reflexos orbiculares-reniformes. Pínnulas cuneado-flabeladas. . . . . . Adiantum L.

9'. Soros nunca insertos sobre los márgenes reflexos. Últimas divisiones de la lámina de otras formas . . . . . . . (10)

10. Frondes palmatipartidas $\ldots \ldots \ldots \ldots \ldots \ldots \ldots \ldots \ldots \ldots \ldots \ldots \ldots \ldots \ldots \ldots \ldots \ldots \ldots \ldots \ldots \ldots \ldots$ Doryopteris.

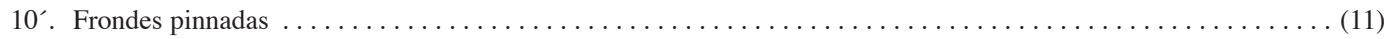

11. Pecíolos con dos expansiones laterales aliformes. Esporangios agrupados en soros aislados dispuestos en las termina-

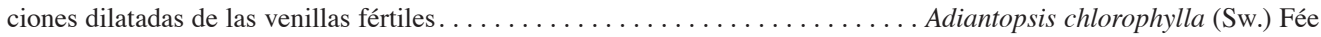

11'. Pecíolos sin expansiones laterales aliformes. Soros contínuos agrupados en cenosoros lineares dispuestos sobre venas colectoras, que dejan libre las porciones apicales de los segmentos fértiles . . . . . . .......Pteris deflexa Link.

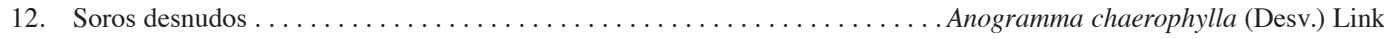

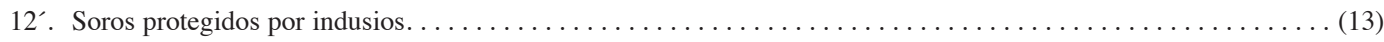

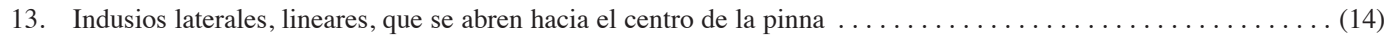

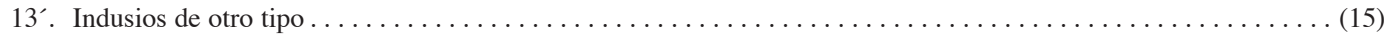

14. Soros alargados, dispuestos en la porción media de las nervaduras secundarias, oblicuos a la costa. Raquis gemíferos

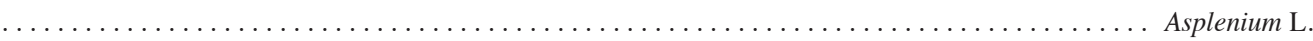

14'. Soros continuos agrupados en cenosoros lineares dispuestos sobre venas comisurales y paralelos a la costa. Raquis no

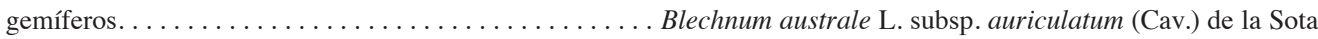

15. Láminas pinnadas. Pinnas sésiles, linear elípticas de ápice redondeado y margen dentado-crenado. Soros con indusios

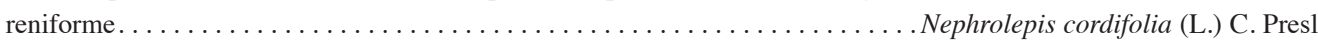

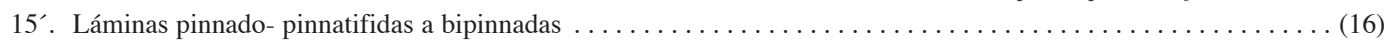

16. Rizoma erecto u oblicuo. Pelos de los ejes rojizos, cortos, finos y romos. Láminas pinnado-pinnatifidas, bipinnatifidas

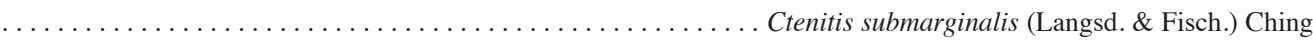

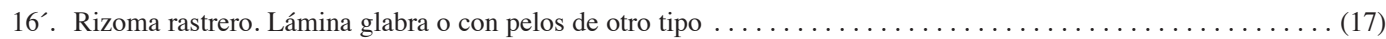

17. Láminas glabras. Con indusio peltado . . . . . . . . . . . . . . . Rumhora adiantiformis (G. Forst.) Ching

17’. Láminas con pelos glandulares capitados y no glandulares setiformes. Soros desnudos o con indusio reniforme piloso Thelypteris Schmidel.

\section{CLAVES DE ESPECIES}

\section{Adiantum}

1. Pinnulas flabeladas de base predominante cuneado- simétrica. Margen de pínnulas dentado, venas terminando en los dientes . . . . . . . . . . . . . . . .

1'. Pinnulas flabeladas de base predominante cuneado- asimétrica. Margen de pinnulas dentado, venas terminando entre

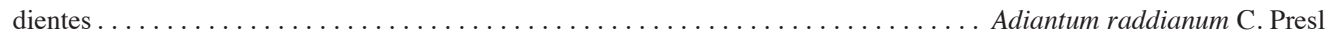

\section{Doryopteris}

1. Láminas con nerviación libre. Pecíolo alado o surcado.......... Doryopteris concolor (Langsd. \& Fisch.) Kuhn

1'. Láminas con nerviación parcial o totalmente areolada. Pecíolo nunca alado o surcado . . . . . . . . . . . . . Doryopteris pentagona Pic. Serm.

\section{Asplenium}

1. Pecíolos hasta $1 \mathrm{~cm}$ de longitud. Pinnas romboidales, casi isodiamétricas, margen serrado

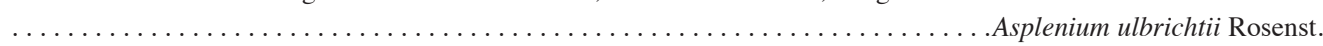

$1^{\prime}$. Pecíolos mayores a $2 \mathrm{~cm}$ de longitud. Pinnas alargadas, falcadas hacia arriba, margen dentado $\ldots \ldots \ldots \ldots \ldots \ldots \ldots \ldots \ldots \ldots \ldots \ldots \ldots \ldots \ldots \ldots \ldots \ldots \ldots \ldots \ldots \ldots$ Asplenium sellowianum (Hieron.) C. Presl ex Hieron.

\section{Thelypteris}

1. Soros desnudos. Pelos 2-5 ramificados, en raquis y costa adaxial. Yemas proliferas en la axila del primer par de

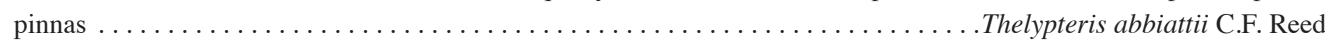

$1^{\prime}$. Soros con indusio reniforme piloso. Cara abaxial glandulosa-pilosa, pelos no glandulares aciculares 1-celulares y pelos

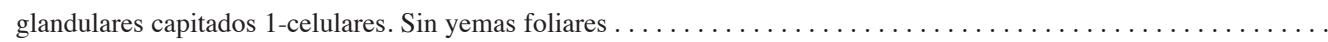


Pleopeltis

1. Lámina pinnada. Cara abaxial cubierta de escamas peltadas acuminadas; cara adaxial glabra $\ldots . \ldots \ldots \ldots \ldots \ldots$ Pleopeltis squalida (Vell.) de la Sota

1'. Lámina entera de margen sinuado. Cara abaxial con escamas peltadas de forma variada, nunca acuminada; cara

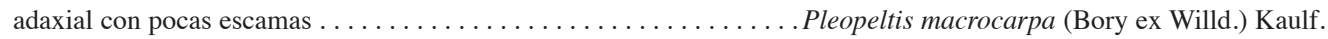

Salvinia

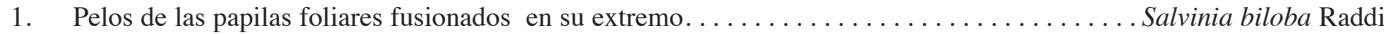

$1^{\prime}$. Pelos de las papilas foliares libres en su extremo $\ldots \ldots \ldots \ldots \ldots \ldots \ldots \ldots \ldots \ldots \ldots$ Salvinia minima Baker

\section{DISCUSIÓN}

Según los datos aportados por de la Sota (1967, 1973), Ponce et al. (2002) y de la Sota et al. (2004) existen tres centros de diversidad de helechos y licofitas en el Cono Sur de America meridional: Noroeste Argentino, Brasil austrooriental y los Bosques andino-patagónicos. En este sentido la flora de helechos y licofitas de la ribera del Río de La Plata muestra una marcada influencia austro-brasilera. Así, los ríos Paraná y Uruguay representarían importantes rutas migratorias fluviales a lo largo de las selvas en galería.

Se infiere que la Isla Martín García, ubicada en el Delta del Río de La Plata $\left(34^{\circ}\right.$ $11^{\prime} 09^{\prime \prime}$ S y $58^{\circ} 15^{\prime} 09^{\prime \prime}$ W) a $46 \mathrm{~km}$ de la ciudad de Buenos Aires, con 170ha de superficie y alrededor de 30 taxa de helechos y licofitas (Lahitte \& Hurrel 1996, Ramos Giacosa com. pers.), constituiría una estación intermedia en la ruta migratoria fluvial, se evidencia un empobrecimiento de la diversidad hacia el sur de la ribera platense, en cuyo límite austral se halla la Reserva Punta Lara.

La presencia de un número reducido de taxa de distribución Andino-pampeana (como por ejemplo Rumohra adiantiformis, Anogramma chaerophylla, Blechnum australe L. subsp. auriculatum, Equisetum giganteum y Adiantum raddianum) pone en evidencia la escasa vinculación florística andino-serrana con la Reserva.

En el caso de Adiantopsis chlorophylla, fue citado por primera vez en la Reserva por Moschione (1987), quien mencionó la presencia de pequeñas poblaciones con individuos jóvenes creciendo en suelos relativamente secos, en una zona de poca accesibilidad. Según nuestras observaciones, los escasos individuos documentados indicarían una disminución de las poblaciones en los últimos años.

Una de las especies más vulnerables en esta zona es Adiantum raddianum debido a que, por su alto valor ornamental, ha sido extraída sin control por recolectores furtivos durante las décadas del 80 y 90, para su posterior comercialización.

Lo mismo sucede con Rumohra adiantiformis, una especie frecuente en las Sierras de Tandil y Balcarce, provincia de Buenos Aires, lugares de donde se están extrayendo con fines ornamentales. La presencia de este taxón en la Reserva fue citada por Moschione (1987, "tres ejemplares" según el autor), hasta el presente se han detectado escasos individuos.

El hallazgo de Doryopteris pentagona en la Reserva Punta Lara, una especie de distribución disyunta y característica del Nordeste y Noroeste argentino podría deberse a una extensión de su área desde el Nordeste de Argentina, a través de cursos de agua del estuario del Plata.

Con respecto a otros taxa citados para esta área en trabajos anteriores y del que no se cuenta con material de referencia, como Osmunda regalis L. var. spectabilis (Willd.) A. Gray y Pilularia americana A. Braun (especie acuática/palustre), éstos no fueron registrados en el período de observación. Se presume que estas especies, poco llamativas desde el punto 
de vista ornamental, podrían haber sido afectadas por fluctuaciones climáticas (periodos de sequía prolongados), las cuales no les permitieron perpetuarse. Moschione (1987) señaló que, debido a la escasez de individuos Osmunda regalis var. spectabilis en la Reserva, su presencia futura era incierta.

Durante el período de observación no se hallaron individuos del género acuático Salvinia. Dado que crecen en cuerpos de agua temporarios, se infiere que los períodos de sequía registrados en la región en los últimos años pueden haber afectado a estas poblaciones.

En el caso de Marsilea ancylopoda (especie acuática/palustre), se hallaron poblaciones conspicuas en zonas destinadas al pastoreo dentro de la Reserva Punta Lara, lo cual indicaría que ésta especie sería más resistente a los períodos de sequía. Además, se identificaron dos especies introducidas no mencionadas hasta el momento: Pteris deflexa y Adiantum capillus-veneris, las que se suman a otras ya citadas por Moschione (1987) como Nephrolepis cordifolia y Pteris vittata. Esta última no fue encontrada en el período de observación.

Las principales causas de amenaza o ausencia de especies de helechos y licofitas en la región estarían dadas por: la extracción para su comercialización, la introducción de plantas exóticas y las fluctuaciones climáticas (períodos de sequía). Otra problemática a tener en cuenta en futuros estudios es la influencia de la contaminación del suelo y del agua por basurales en las zonas aledañas a la Reserva sobre las poblaciones vegetales. Por lo tanto, se tiene prevista la implementación de técnicas de cultivo in-vitro de esporas y desarrollo de gametofitos, como una estrategia para la conservación de las especies de helechos y licofitas más vulnerables en la Reserva Punta Lara, con el propósito final de obtener individuos para repoblar el ambiente natural.

\section{AGRADECIMIENTOS}

Los autores desean expresar su agradecimiento al personal Guardaparques de la Reserva Natural Punta Lara por su continua colaboración durante la realización de este trabajo, a la Fundación de Historia Natural Félix de Azara por su apoyo a los proyectos de Conservación de la Flora Argentina y a Elián Guerrero por su permanente colaboración en la recolecta de material. Este trabajo se realizó con subsidios de UNLP (11N/465) y CONICET (PIP 878).

\section{RESUMEN}

Hasta el año 1987 el número de helechos y licofitas citados para la Reserva Punta Lara era de 30, incluyendo taxa específicos e infraespecíficos. El objetivo de este trabajo fue evaluar la diversidad de los helechos y licofitas que crecen en esta reserva, donde se halla el relicto más austral de las selvas en galería. Para llevar a cabo este estudio se realizaron viajes de campo durante los años 2006-2009 y se consultaron herbarios locales así como bibliografía especializada. Como resultado del estudio se determinaron 24 taxa de helechos y licofitas, de hábito predominantemente terrestre (70\%); pocas acuáticas (17\%) y epífitas (13\%); dos especies exóticas, mientras que otras dos especies nativas citadas previamente no fueron halladas. Cinco taxa fueron registrados con escasa frecuencia. Se infiere que las principales causas de amenaza para las poblaciones de licofitas y helechos en la Reserva Natural Punta Lara son la extracción para su comercialización, la introducción de plantas exóticas y las fluctuaciones climáticas (periodos de sequía). Se presenta una clave de identificación de los taxa registrados y se plantean futuras estrategias de conservación de las especies consideradas vulnerables.

Palabras clave: helechos, licofitas, Argentina, Buenos Aires, Reserva Natural Punta Lara, diversidad.

\section{REFERENCIAS}

Cabrera, A.L. \& G. Dawson. 1944. La selva marginal de Punta Lara en la ribera argentina del Río de La Plata. Revista Mus. La Plata. Secc. Bot. 22: 167-305.

Cabrera, A.L. 1960. La selva marginal de Punta Lara. Ciencia e Investigación 16: 439-446.

Cabrera, A.L. 1971. Fitogeografía de la República Argentina. Bol. Soc. Argent. Bot. 14: 1-42.

Cabrera, A.L. 1976. Regiones fitogeográficas argentinas. Enciclopedia Argentina de Agricultura y Jardinería. Buenos Aires, Argentina.

Capurro, R.H. 1969. División Pteridophyta, p. 123-146. In A.L. Cabrera (ed.). Flora de la Provincia de Buenos 
Aires. Colecc. Ci. Inst. Nac. Tecnol. Agropecu. 4: 123-146.

Dascanio, L.M. \& S.E. Ricci.1988. Descripción florísticoestructural de las fisonomías dominadas por árboles en la Reserva Integral de Punta Lara (Pcia. de Buenos Aires, República Argentina). Revista Mus. La Plata, Secc. Bot. 14: 191-206.

de la Sota, E.R. 1967. Composición, origen y vinculaciones de la flora Pteridológica de las sierras de Buenos Aires (Argentina). Bol. Soc. Argent. Bot. 11: 105-128.

de la Sota, E.R. 1973. La distribución geográfica de las Pteridofitas en el Cono sur de America meridional. Bol. Soc. Argent. Bot. 15: 23-34.

de la Sota, E.R., G.E. Giudice, M. Ponce, J.P. Ramos Giacosa \& M. Arturi. 2004. Relaciones fitogeográficas de la flora pteridofitica serrana bonaerense. Bol. Soc. Argent. Bot. 39: 181-194.

Delucchi, G. 2006. Las especies vegetales amenazadas de la Provincia de Buenos Aires: una actualización. APRONA Bol. Cient. 39: 19-31.

Holmgren, P.K., N.H. Holmgren \& L.C. Barnett. 1990. Index Herbariorum. Part I: The herbaria of the world. New York Botanical Garden, Nueva York, EEUU.
INDEC 2001. Censo Nacional de Población. Instituto Nacional de Estadísticas y Censo. Buenos Aires, República Argentina.

Lahitte, H.B. \& J.A. Hurrell. 1996. Planta hidrófilas de la Isla Martín García. Comisión de Investigaciones Científicas (CIC) Buenos Aires.

Mickel, T.J. 1977. Rare and endangered Pteridophytes in the New World and their propects for the future, $\mathrm{p}$. 232-230. In G.T. Prance \& T.S. Elias (eds.). Extinction is forever. The New York Botanical Garden, Nueva York, EEUU.

Moschione, F.N. 1987. Ampliación de la distribución de seis especies de helechos (Pteridophyta, Filicopsida) en la ribera Argentina del Río de La Plata. Garganchillo 1: 7-13.

Ponce, M., K. Mehltreter \& E.R. de la Sota. 2002. Análisis biogeográfico de la diversidad pteridofítica en Argentina y Chile continental. Rev. Chil. Hist. Nat. 75: 703-717.

Zuloaga, F.O., O. Morrone \& M. Belgrano. 2008. Catálogo de las plantas vasculares del Cono Sur. Vol. 1, Pteridophyta, Gymnospermae y Monocotyledonae. Monogr. Syst. Bot. Missouri Bot. Gard. 107. 